\title{
GLAD!
}

Revue sur le langage, le genre, les sexualités

05 | 2018

Raconter les sexualités depuis la marge

\section{Ce qui reste : enquête et écriture sur un territoire marginal}

What's Left: Inquiry and Writing on a Marginal Territory

\section{Magdalena Brand}

\section{(2) OpenEdition}

\section{Journals}

Édition électronique

URL : http://journals.openedition.org/glad/1190

ISSN : 2551-0819

Éditeur

Association GSL

\section{Référence électronique}

Magdalena Brand, « Ce qui reste : enquête et écriture sur un territoire marginal », GLAD! [En ligne], 05 |

2018, mis en ligne le 15 décembre 2018, consulté le 17 décembre 2020. URL : http://

journals.openedition.org/glad/1190

Ce document a été généré automatiquement le 17 décembre 2020.

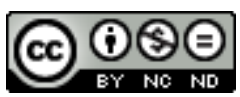

La revue GLAD! est mise à disposition selon les termes de la Licence Creative Commons Attribution Pas d'Utilisation Commerciale - Pas de Modification 4.0 International. 


\section{Ce qui reste : enquête et écriture sur un territoire marginal}

What's Left: Inquiry and Writing on a Marginal Territory

\section{Magdalena Brand}

Femme des eaux

Je te salue

Que ne sècherai-je pas la mer

Pour retrouver la trace

Des sœurs parties

Vers une destinée inconnue

Sans nos bénédictions

Sans nous dire adieu

Tsibinda $1987: 30-31^{1}$

Un fleuve c'est peut-être des larmes, des larmes qui

coulent ensemble

Yabouza $2008: 166$ 


\section{Introduction}

D'abord c'est une minute de ce silence pour ce quartier, parce qu'avant il y avait toute une vie là. Et maintenant, c'est un grand calme qui règne, un grand silence. Et toute la sueur des gens qui ont laissé leur marque, leurs traces de pas, leur passage sur ce quartier. Cela me donne une grande émotion de remarcher encore une fois de plus sur notre parcours, notre petit quartier et cette joie des gens qui vivaient là. Ils ne sont pas oubliés. [...] J'espère que là où ils sont tous, ils retrouvent la paix, et le soutien de leur famille, ainsi que la joie dans leur cœur. Moi-même, j'avais peint un cœur ici, mais je ne savais pas que d'ici trois ans plus tard il resterait là après toutes ces années. [...] Comme ce symbole, malgré là où on est dans les différents coins, d'où nous nous trouvons, on gardera toujours ce même lien, cette amitié, qui sont des liens forts et un grand moment de partage, comme une vague d'eau au bord du fleuve Oubangui. (Jean, 2016)

1 Deux ans après la soutenance de ma thèse, je reçois cet enregistrement audio-visuel. Il m'a été envoyé à Paris depuis la capitale centrafricaine, Bangui, par un des personnages importants de mes recherches. Jean habitait avec sa sœur le quartier où j'ai conduit mes enquêtes. Un quartier où les femmes étaient chefs de famille. Les autres habitante-s de la ville les appelaient les «femmes libres». Une de mes voisines au quartier, m'avait donné à peu près cette définition : « tellement elles ont usé de leur liberté, c'est devenu péjoratif ». Pour vivre, elles partaient travailler chaque nuit dans les espaces de loisirs des expatriés (les bars, les restaurants et les boîtes de nuit du centre-ville) comme prostituées et serveuses. À l'époque de mes enquêtes, Jean était artiste peintre de métier et homme à tout faire dans une organisation internationale pour gagner sa vie. Le soir et la nuit, il gardait les enfants pendant que sa sœur partait travailler au Casino comme croupière. Aujourd'hui, il fait des allers-retours entre les camps de réfugiés au Congo et Bangui pour survivre à la guerre qui a fait disparaître ses moyens de travail et qui a détruit son quartier. Ses ancien-ne-s voisin-e-s se sont réfugié-e-s dans d'autres quartiers plus éloignés du centre-ville et des combats et dans d'autres pays frontaliers pour trouver du travail. Jean a filmé les marques de leur passage. Jean a documenté les traces. Je regarde encore et encore ces images: les restes des murs, le tracé de l'eau qui s'écoulait au milieu du quartier, les tâches laissées par les feux des cuisines. La disparition des foyers. La dispersion des liens. Jean a documenté la perte. Je me rends compte que j'écris encore des articles faisant usage du temps présent pour décrire un quartier qui n'existe plus aujourd'hui. Quel est le rapport de l'écriture ethnographique avec la perte?

2 Dans cet article, je souhaite réfléchir aux enjeux de l'écriture après une enquête ethnographique sur un territoire urbain marginalisé et stigmatisé. Cette réflexion fait suite à une recherche de terrain sur les «échanges économico-sexuels» (Tabet 2004) entre des expatriés français et des femmes centrafricaines à Bangui. Mon but était de démontrer « la grande arnaque » (Tabet 2004) : de montrer que l'expatriation française à Bangui reposait sur un travail sexuel dévalorisé et invisibilisé de femmes centrafricaines stigmatisées.

3 Certains moments de l'enquête orientent le processus de connaissance théorique qui mène à l'écriture. Pour moi, ce fut une nuit, sur la route du retour entre le bar où travaillait une de mes voisines, Andrea, et le quartier. Alors que nous étions en train de marcher, Andrea me dit qu'elle n'en peut plus de faire des choses qu'elle n'a pas envie 
de faire, que des fois elle ne se reconnait plus elle-même. Je me souviens que je n'ai rien dit. J'ai gardé le silence. J'avais un sentiment de désespoir et je crois bien qu'Andrea a perçu ma gêne. Le lendemain, Andrea vint me voir chez moi et me dit à peu près ceci : "attention, ne crois surtout pas que je suis soumise à ce type, je sais pourquoi je fais tout ça, j'ai mes projets et ça c'est ma liberté ». Ce moment s'inscrivait dans la vie quotidienne du quartier où les relations personnelles de voisinage permettaient de revenir le lendemain sur des sentiments de la veille, donnaient l'occasion non pas seulement d'expliquer mais de s'expliquer. Ces relations personnelles quotidiennes que j'ai pu vivre au quartier m'ont apporté la certitude qu'une relation pour de l'argent avec un homme ne veut pas dire que celui-ci possède le corps, la personnalité ou la sexualité d'une femme qui investit du temps et de l'argent dans une sexualité et une féminité de service. J'avais prêté le livre La grande arnaque de Tabet (2004) à Andrea. Andrea partageait son temps entre sa maison au quartier et la maison d'un de ses copains. Elle m'a dit qu'elle voulait laisser le livre dans sa " petite cabane ", pour ne pas que son copain le voie. Elle l'a enfoui dans ses vêtements en disant, « sinon, il va savoir que je l'arnaque et que je sais qu'il m'arnaque ». Le quartier était bien le lieu où la théorie, au sens de la conscience et de la maîtrise sur la vie avait la possibilité d'exister. Mais une nuit, l'installation électrique précaire a mis le feu au double toit en carton de la chambre d'Andrea et le livre La grande arnaque a brûlé. Andrea a préféré quitter le quartier définitivement pour partir vivre à plein temps chez son copain.

Les liens entre la vie quotidienne et la théorie sont tissés au jour le jour et dépendent des espaces d'autonomie et des possibilités matérielles de circuler entre des espaces ségrégués, de garder des traces et de sauvegarder des relations sociales. L'objectif de cet article est d'interroger le passage entre l'enquête et le texte : que reste-t-il de la vie quotidienne disparue? L'écriture ethnographique peut-elle se faire la trace de ces rencontres précaires entre la vie quotidienne et la théorie? Quelle est la perte dans le texte ethnographique?

5 Pour réfléchir au rapport entre l'écriture ethnographique et la perte, je présenterai une par une trois figures de mon écriture : le quartier, Mami-Wata et Ballerine. Le quartier était un territoire de transmission de mots de résistance, un espace de pratiques et de savoirs. Le texte ethnographique, qui se déplace d'un contexte de discours à un autre, du quartier à l'université, peut-il se faire la trace de cet entrelacement de la vie quotidienne et de la théorie ? Comment transmettre ici ce qui s'est dit ailleurs? MamiWata est un esprit de l'eau apparu en Afrique centrale en même temps que la colonisation. Elle possédait certaines femmes métisses au quartier. De quoi Mami-Wata est-elle la trace? Qu'est-ce qui se perd dans l'écriture académique de Mami-Wata? Ballerine était une association de femmes prostituées ayant existé dans les années 90. Tout comme le quartier, Ballerine a disparu et se loge dans les mémoires des ancien-nes habitant-e-s. Comment écrire la perte? Que dit-elle?

\section{Le quartier}

6 C'était un petit quartier caché en plein milieu du centre-ville, derrière de grandes maisons à étages et un restaurant. Il se trouvait derrière l'ancienne Avenue Gentil, l'ancien quartier des Européens. Sur une carte postale du début du XXe siècle que j'avais trouvée chez un collectionneur, j'y ai vu des colonisés travaillant au défrichage $\mathrm{du}$ terrain pour la construction des maisons coloniales, sous le regard du photographe 
qui était visiblement aussi le contrôleur des travaux. Aux jours de mon enquête, le quartier était une grande concession divisée en une dizaine de maisons. Les habitant-e$\mathrm{s}$ du quartier appartenaient à des collectifs de travail avoisinants qui se soutenaient au quotidien: les lavandières et blanchisseuses des hôtels, les gardiens des hôtels, les cuisiniers de restaurant, les sécurités des boîtes de nuit, les serveuses, les danseurs et les ambianceurs, les femmes qui marchent la nuit. Jean les appelait les «BoxerBangui », celles et ceux qui survivent là où les plus riches et les plus blancs s'amusent. Le quartier était situé géographiquement au cœur de la ville, mais socialement loin des autres habitant-e-s. Jean m'avait expliqué : «C'est étrange parce que d'autres ignorent l'existence de ce petit quartier. (...) Ils ignorent que des gens vivent ici. Tu vois on est entouré de tous les beaux immeubles». Si les expatriés-e-s ignoraient la vie quotidienne du quartier, cela n'était pas dû au hasard. Les habitant-e-s ne laissaient aucun homme étranger rentrer dans le quartier sans l'accord préalable des femmes. Le seul expatrié français que j'ai rencontré qui connaissait l'existence du quartier l'appelait la cour des miracles: " là où vivent les putes et les godobès ${ }^{2}$ ». Les termes stigmatisants utilisés pour qualifier le quartier indiquent l'ignorance de l'organisation familiale qui rendait l'existence de ce dernier possible.

7 Les murs tenaient une dizaine de familles. Les femmes en étaient les premières génératrices de revenus. Elles les gagnaient dans le travail informel du sexe et dans le travail de service domestique et sexuel dévalorisé, dans les hôtels et les restaurants du centre-ville. « Les femmes ici refusent le travail des hommes » m'avait expliqué une fois Jean, ce qui voulait dire refuser de dépendre du salaire d'un homme. Les hommes qui habitaient la concession étaient leurs frères et leurs enfants, ou des hommes plus pauvres qu'elles et à qui elles déléguaient un travail. Les hommes avec qui elles avaient des relations de dépendance économique n'habitaient pas le quartier. Les femmes décidaient de leur présence à certaines occasions précises, selon ce qu'elles voulaient faire. Je me souviens, comment, le jour de mon anniversaire, ma voisine Laetitia, la sœur de Jean, m'a invitée à sortir et m'a dit de venir chez elle pour m'habiller. On avait rendez-vous avec un militaire au " Grand Café ", une boulangerie du centre-ville. Avant de sortir, je lui avais demandé si je devais prendre de l'argent avec moi. Elle m'avait alors pris le visage des deux mains pour me dire en riant: «Ma chérie, c'est gratuit pour toi ce soir, il a le droit ${ }^{3}$ de payer pour toi ce soir. C'est pas grave. Tu verras, tous, ils vont payer pour toi. » Je découvrais que les femmes ne sont pas seules dans l'espacetemps de la nuit mais s'organisent à plusieurs.

8 La source d'eau du quartier unissait les différents foyers. Jean avait expliqué pendant l'entretien :

Dans cette petite source, tout le monde vient puiser de l'eau dedans. Quand c'est bouché, c'est tout le monde qui se cotise de l'argent. Personne ne refuse de cotiser. Souvent c'est Adèle, ou bien Simone, ou bien ma sœur qui prennent leur courage et elles le font pour les autres. La personne dit qu'elle ne nous aime pas. Mais au fond tu te rends compte que non. Si la personne ne nous aimait pas, pourquoi elle aurait creusé un truc pour tout le monde, elle aurait dû faire un truc pour chez elle. Moi je sais que c'est comme ça, chacun est chez soi, mais il y a l'amour de l'autre qui est là. Parce qu'on a tous le même sang. Je ne vois pas de différence. (Jean, 2010)

9 C'était Tantine Eyabe qui en était la gardienne. Contre le paiement de 100 CFA par sceau, elle ouvrait le robinet d'eau avec la clef qu'elle avait autour du cou. Même en cas 
de conflits entre les différentes unités familiales du quartier, Tantine Eyabe était respectée et considérée par tous les protagonistes. Elle connaissait chaque personne, ses problèmes et ses solutions. C'est l'expérience de Tantine Eyabe en tant que femme dans la ville de Bangui qui faisait d'elle la doyenne du quartier. Elle m'avait expliqué avoir décidé de prendre sa «retraite des hommes ». Elle était la source de nombreux conseils. Elle faisait du quartier un lieu de transmission. Maman Rosette, retraitée comme Tantine Eyabe, transmettait elle aussi. Elle me disait: "Ma fille, tu devras toujours négocier de façon stratégique. Il ne faut pas dire les choses directement ni brutalement sinon tu seras renvoyée.» Le vocabulaire de la ruse était souvent convoqué dans les discussions de mes voisines.

10 Comme par ruse, le quartier n'avait pas de nom officiel. Certaines femmes l'appelaient le village. Jean et les autres frères du quartier lui avait donné deux noms : Soweto, comme le township d'Afrique du Sud qui s'est révolté en 1976 contre le pouvoir blanc ; et Quartier latin, en référence au groupe musical du Congo-Kinshasa dont a fait partie Koffi Olomide. Jean m'avait expliqué :

Il fallait un nom au quartier. Normalement il n'y a pas de nom, c'est juste un petit quartier. (...) D'autres l'appellent le village ou encore Soweto. Parce qu'il n'y a pas un nom précis. Les gens n'ont pas cherché à nommer ce quartier parce que tout le monde pense qu'ils sont ici provisoirement. Du jour au lendemain, on peut vous dire, voilà c'est fini, il faudrait que tout le monde parte d'ici. (Jean, 2010)

11 Jean a cherché à nommer son quartier en prévision de sa disparition, pour en garder les traces. Tout au contraire de sa démarche, l'écriture ethnographique cherche à anonymiser. L'écriture ethnographique ne nomme pas, l'écriture ethnographique veut qualifier. Comment qualifier sans nommer? Comment qualifier dans le contexte académique ce qui a été nommé ailleurs ? Qu'est-ce qui se perd avec la disparition des noms?

12 La première fois que j'ai raconté les récits de Jean et de sa sœur Laeticia dans une cafétéria de l'université française, on m'a répondu : «ah, mais son frère est son mac ». Le bruit de l'eau distribuée qui coule, la chaleur des corps, le silence de la nuit quand les femmes sont rentrées bredouilles, la lumière du petit matin à l'heure où les frères partent travailler comme homme à tout faire, tout cela avait disparu. La disparition des noms c'est la disparition des attachements, des affects et des liens qui rendent possible la reconnaissance. Judith Butler s'est interrogée sur cette question des «cadres à travers lesquels nous appréhendons ou au contraire échouons à appréhender les vies des autres ». Dans son livre, Ce qui fait une vie, (Butler 2010) elle se demande comment créer une pensée de la reconnaissance, alors que les cadres, dans lesquels les lecteurs voient, pensent et agissent, opèrent de sorte à produire certains sujets reconnaissables et d'autres "infiniment plus difficiles à reconnaître ». L'écriture académique fait voyager de force des vies d'un contexte à un autre : elle change les noms et perd les liens d'attache qui rendaient une vie reconnaissable parmi les autres.

\section{Mami-Wata}

Les mami watas, les mauvais esprits, ils veulent coucher avec moi. Ce sont quatre hommes. Des blancs. Quand je dors, ils viennent. Quand ils viennent, ça bouge. Les parfums et les crèmes tombent. Je sais que ce sont eux qui 
viennent pour me voir. Ils sont venus avec des plats de riz pour me donner à manger. Mais je refuse. Je dis non, je ne veux pas manger votre repas. Je pleure, je crie. Alors ils partent.

Les blancs sont gentils. Ils veulent seulement coucher avec moi, ils veulent que je devienne leur femme. Je dis non je ne veux pas! Ils veulent encore ! Ils imposent que je devienne leur reine. Je refuse. Ils m'ont apporté des couronnes de couleur rouge. J'ai refusé. Ils m'ont donné des fleurs roses.

Les quatre hommes blancs m'ont dit de tuer ma mère et de tuer Jean, mon mari. C'est comme si ma mère avait beaucoup prié, et Jean avait beaucoup prié. Ils m'ont donné un couteau, un gros couteau, ils l'ont mis sur mon cœur, ils ont dit de tuer ma mère, ils m'ont dit de tuer Jean. J'ai dit non je ne peux pas. Ils ont dit si je refuse, je dois mourir. J'ai dit « oui j'accepte, je crois à mon Dieu, je ne peux pas mourir.» (Elisabeth, 2010)

13 Elisabeth était la copine de Jean. À l'époque de l'entretien, elle avait une vingtaine d'années et venait d'emménager dans la parcelle de Laeticia, la sœur de Jean. J'ai assisté à une de ses crises de possession. Prise de peur, j'ai voulu partir. On m'a demandé de rester. Pourquoi ai-je eu peur? En tant que femme blanche, je sentais que je faisais partie de cette histoire de possession et je me sentais en danger: j'avais peur que l'agressivité d'Elisabeth se tourne contre moi. À mon retour en région parisienne, j'ai très vite été fascinée par les rappels répétitifs de cette figure de Mami-Wata rencontrée durant mes enquêtes au quartier. J'avais l'impression de la retrouver dans des articles, des archives et des romans. Au retour de mon premier terrain, je suis tombée un peu par hasard sur un article écrit par Michel Ogrizek, un médecin ayant pratiqué dans la région de Batsangui au Congo à la fin du XXe siècle. Dans son article intitulé «Mami Wata, les envoûtées de la sirène. Psychothérapie collective de l'hystérie en pays Batsangui au Congo, suivie d'un voyage mythologique en Centrafrique " (Ogrizek 1981-1982), il fait le récit de sa recherche sur le parcours thérapeutique de soins pour les femmes envoutées par Mami-Wata et de son trouble pendant une séance de désenvoûtement :

On ne peut être un spectateur neutre à un psychodrame. Il me faudrait analyser mon propre trouble lors de la séance, mon impression qu'il se passait quelque chose (mais quoi ? mais où ?) (Ogrizek 1981-1982: 439)

La problématique était la blancheur de mon teint: certains craignaient une agressivité de la patiente à mon égard. Tout au long de la séance, la féticheuse prit soin de se placer entre l'envoûtée et moi-même chaque fois que cette dernière se rapprochait trop de l'endroit où j'étais assis. (Ogrizek 1981-1982 : 437)

14 À la lecture du texte du médecin, Mami-Wata me permettait d'éclairer les situations et les interactions traversées par l'histoire coloniale. Elisabeth est la fille d'un enfant métis non reconnu par son père, un colonel de l'armée française, qui a abandonné la mère de l'enfant pour retourner en France où il fonda une famille avec une femme blanche de sa classe sociale. J'étais la potentielle épouse blanche légitime de l'homme blanc. Je faisais partie du psychodrame pour reprendre les termes du médecin. Dans mon trouble se rejouait un autre trouble : l'histoire coloniale.

15 En 2012, sachant que je ne repartirai plus à Bangui, je me suis mise à la recherche d'archives historiques. J'ai alors retrouvé Mami-Wata chez un collectionneur. Didier Carité est un ancien expatrié, coopérant français et collectionneur depuis des dizaines d'années. C'est dans le grenier d'une maison de la région parisienne qu'il me présenta sa collection de cartes postales sur la Centrafrique. Dans un espace transformé en cabinet de curiosités, je découvrais des cartes postales consignées dans des albums et 
des boîtes, rangées par dates et par thèmes. Elles côtoyaient des objets d'un usage passé exposés sur des armoires et illustrant la fabrique du regard européen sur l'Afrique centrale. Didier Carité a rassemblé dans un article les cartes postales coloniales représentant deux danseuses en Oubangui-chari, Hellani et Yakoyé (Carité 2009-2010). Elles ont été les deux modèles principaux de Robert Carmet et Bernard Lefebvre, photographes-reporters au service des forces aériennes françaises libres en AEF, entre 1940 et 1944. Les images de Hellani et Yakoyé, produites dans le contexte colonial des années 1940, ont traversé les époques et franchi les indépendances, se retrouvant dans les publicités et les cartes postales des années 1960 et 1970. La diffusion de l'image n'est pas reliée aux modèles. On décèle là l'Empire analysé par Edward Saïd, qui se reconnaît par son «aptitude à produire certaines images intellectuelles étrangement autonomes» (Saïd 2000 : 173). Face à la folie apparente d'une telle reproduction déconnectée de la vie, le récit du collectionneur a invoqué Mami-Wata pour donner sens à sa collection : "vos images qui subsistent, grâce à Bernard et à Robert, ne vous ont-elles pas plutôt transformées en admirables et éternelles Mami Wata oubanguiennes? » (Carité 2009-2010 : 19) Mami-Wata a pour effet de dématérialiser le travail de Hellani et Yakoyé, de les détacher du contexte historique et de l'interaction à la base des photographies. En invoquant Mami-Wata, Didier Carité a transformé Hellani et Yakoyé en images et les a insérées de force dans l'imaginaire de sa collection: Hellani et Yakoyé sont devenues des objets de l'imaginaire colonial, et non plus sujets de leurs vies dans un contexte donné.

Joseph Tonda est anthropologue et sociologue spécialiste de la culture gabonaise et congolaise, il est professeur de sociologie à l'Université Omar Bongo à Libreville et chercheur à l'École des hautes études en sciences sociales à Paris, en France. Dans son livre, Le Souverain moderne, le corps du pouvoir en Afrique centrale (Tonda 2005), Joseph Tonda s'interroge sur ce que cette divinité fait aux corps. Mami-Wata est une des dimensions de la modernité, une des incarnations du «Souverain moderne » qui fait violence aux corps en les organisant autour des choses du blanc. La magie ici, ce sont les fétiches des blancs (miroir, parfums, bijoux...) qui symbolisent l'individualisation extrême et qui possèdent les corps des colonisées. Mami-Wata dans le texte anthropologique permet de raconter l'histoire coloniale et la puissance de la dépossession sur le plan matériel et de l'imaginaire. Les forces sociales mises en scène par Joseph Tonda à travers la figure de Mami-Wata sont patriarcales : l'ordre moral masculin précolonial et l'ordre capitaliste et religieux colonial. Mami-Wata, la modernité, est le bouleversement du lignage précolonial dans lequel les femmes étaient des forces de travail au service du lignage. La figure de Mami-Wata telle qu'elle est traduite par les anthropologues fait croire que les femmes du quartier ne font que répéter une condition issue de l'histoire coloniale : elles continuent d'être possédées, elles continuent de travailler pour le plaisir des blancs. Or, au quartier, Mami-Wata s'articule à une possibilité de résistance collective et organisée. 
Alors, j'ai mes crises de nerfs. Quand j'entends les militaires français, je suis tentée, je me prépare pour les rejoindre, je m'habille comme elles, je me maquille comme une gba munju ${ }^{4}$, je me prépare pour les rejoindre. Mais je résiste. C'est vrai que tu vas prendre des sommes, mais tu peux tomber malade. Le sida. Et puis ils ne voudront jamais de toi comme femme. Tu peux recevoir 50000 par jour. Mais après, les dépenses que tu auras sur ton corps, pour la nourriture et le loyer, il te reste rien. J'ai pensé que c'est dangereux. Mais j'ai pensé aussi... Les filles qui font ça, je les trouve très courageuses. Pour le faire il faut avoir le courage. (Nadège, 2011)

17 Nadège était âgée d'une trentaine d'années à l'époque de mes enquêtes. Elle ne travaillait pas dans les restaurants et les boîtes de nuit. Elle est l'enfant d'une femme libre et d'un expatrié. Elle aurait dû rejoindre la famille de son père après la mort de ce dernier, c'était une de ses promesses. Mais quand je l'ai interviewée, cela faisait plus de dix ans qu'elle attendait toujours. Elle vivait seule avec son fils, dont le père était parti en France sans lui laisser de nouvelles. Là encore, Nadège attendait. Comme Laeticia, elle était possédée par Mami-Wata. Là aussi, la possession par Mami-Wata exprimait la continuité de la violence du concubinage colonial. Mais il y avait une autre réalité exprimée. Dans le récit de ses crises, Nadège parlait de sa tentation non pas simplement de rejoindre les blancs, mais de faire comme les femmes "gba munju », celles qui vont avec les blancs. Son récit a rendu visible non pas seulement les sujets du pouvoir, les blancs, mais aussi un autre collectif, les femmes travailleuses. La prétention de ces dernières à exiger des hommes une compensation matérielle contre leur travail sexuel faisait d'elles des femmes courageuses aux yeux de Nadège. Le récit de Nadège peut faire exister une Mami-Wata ambivalente : personnage à la fois de l'accaparement du corps et de sa résistance physique. Une résistance certes réactive et douloureuse, mais qui pourrait faire place à une réponse collective.

18 Cette résistance s'est perdue dans l'écriture académique de Mami-Wata. Elle a sûrement disparu du compte-rendu ethnographique en même temps que mon trouble et mon sentiment d'insécurité. Elle a perdu le visage de celle qui a produit le trouble de l'ethnologue en lui faisant ressentir son propre rôle dans la répétition de l'histoire coloniale.

\section{Ballerine}

Ballerine est une association qui a existé dans le quartier durant les années 1990, pour résister contre les rafles policières et les violences des clients, au moment de l'occupation militaire française de la ville sous couvert de l'opération Barracuda. Son organisation fait écho aux associations de femmes libres ayant existé pendant l'époque coloniale. Ballerine, c'était une organisation de la vie en dehors du travail: une solidarité contre l'invisibilité. Pour cela Ballerine s'est tout d'abord renommée :

Au début c'était "femme libre". Mais après, on a dit non! On ne veut pas. Nous, on veut Ballerine. "Femme libre" ça n'est pas bon, c'est sans considération. Alors que Ballerine, c'est un mot de passe, on est toutes dans la même.

Ballerine ça n'était pas une petite association. Il fallait voir ça! Avant on portait des choses que tu ne peux même pas croire, parce qu'on avait de l'argent. Il y avait des filles qui changeaient la mobylette tous les trois mois. Et puis tu louais les grandes maisons comme ça. Notre association louait 
même une maison. On était avec des grands hommes d'affaires, des diplomates.

Les femmes de Ballerine, on était nombreuses. On avait des voitures, des manteaux. On a toutes voyagé.

Il y en a qui sont mortes.

Ballerine c'était l'association pour s'organiser, pour qu'on puisse rester dignes devant les gens qui sont venus, pour donner des conseils.

Ballerine c'était 1000 Francs par personne pour le fond de caisse, en cas de maladie et d'hospitalisation. On se rassemblait ensemble, on mettait toujours l'argent. Des fois, il y en avait une qui pouvait dépenser la caisse pour sauver l'autre, sans demander l'avis de personne. (Ballerine, 2010)

La solidarité allait jusque dans la mort. Les femmes de Ballerine portaient le deuil de leurs camarades. Elles confisquaient les corps des défuntes aux parents qui refusaient l'argent des prostituées. Elles organisaient une procession publique, elles étaient visibles, elles étaient reconnues : les gens dans la rue s'avertissaient de leur passage. En confisquant et en portant, dans l'espace public, le corps de leurs camarades décédées, et en l'associant à leurs corps encore en vie, les femmes de Ballerine affirmaient la valeur de leur vie :

Le fond de caisse c'était aussi pour les cas de malheur.

Ah! En cas de malheur, pour nous là, personne ne touchait à ça! C'était nous! Même la famille ne s'approchait pas! Il n'y avait que nous, il n'y avait que nous. On s'occupait de tout : cercueil, tout, tout, tout !

"Cotisation!"

On commençait à la place mortuaire et on allait jusqu'au cimetière. Les familles se fâchaient, mais, avec notre tête têtue, elles restaient tranquilles.

On ne quittait pas! Même si les bagarres tombaient. C'était notre amie! C'était notre sœur ! On avait fait la vie ensemble avec elle !

$\mathrm{Tu}$ entendais les gens de quartier qui criaient: "les filles libres! les filles libres!"

Nous on s'en foutait ! Et puis, si le patron était au courant, il envoyait les videurs de la boîte, les portiers, qui nous suivaient derrière, s'il y avait cas de quoi dans le quartier.

Après, on prenait le reste de l'argent et on le donnait à la famille. Si la famille refusait l'argent, on prenait l'argent et on faisait la fête. Il fallait que l'argent termine le même jour! On mettait ça au milieu de la table et on dépensait. On faisait la fête. On achetait du méchoui et de la viande préparée. Nous, on ne voulait pas préparer. On achète. Le poulet grille. Il y a quatre, cinq casiers de bouteilles. On fait la fête! L'argent qui restait encore, on le partageait entre chacune pour rentrer en taxi et c'était fini. (Ballerine, 2010) discours de Mami-Wata qui produit un sentiment de répétition et de fatalité, le discours de Ballerine met en évidence des " évènements de faible intensité » (Farge 2002) qui s'inscrivent dans un temps, un lieu et un collectif spécifique : 
Maintenant, ça a changé, c'est chacune de son côté. Les petites filles, elles font leur vie en cachette. Elles ne veulent pas que les gens sachent, pour que les gens ne les détestent pas dans le quartier. Donc, elles sortent la nuit tard, quand il n'y a plus personne. Maintenant, il y a beaucoup de filles qui se cachent. Elles sont avec les blancs, mais elles ne montrent pas qu'elles font ça, c'est ça qui n'est pas bien. Mais nous on avait Ballerine. C'était notre vie ! On ne cachait rien! Comment tu peux cacher? Où est le problème? Tu cherches à vivre non! (Ballerine, 2010)

La mémoire de Ballerine fait exister la conjoncture dont parle Jean :

C'est grave la conjoncture, c'est ce qu'on vit. La conjoncture, c'est ce que la vie nous ouvre les yeux à voir. Tu vois, nous on est en train de vivre, on cherche à comprendre le monde, essayer de trouver pourquoi les autres ne voient pas comme nous, on cherche à savoir comment, qu'est ce qu'il faut faire, et ce qu'il faudrait faire. Tout ça c'est pour nous emmener à une raison. (Jean, 2010)

23 La conjoncture est indissociable d'un processus de connaissance et de vision, et d'un ancrage dans un lieu spécifique. Le discours de Ballerine permet de faire exister l'histoire du quartier : les possibilités d'une transformation qui n'a pas eu lieu. Ballerine donne une leçon d'écriture : sa force est de porter le deuil, de porter les disparues, de les exposer, de les nommer. Ainsi, elle se fait le récit d'une force sociale et collective propre aux femmes "gba munju». La mémoire de Ballerine garde les noms: elle nomme les disparues, elle garde mémoire, elle fait histoire, elle célèbre une époque et ses protagonistes. C'est une mémoire vivante et donc possiblement transformatrice.

\section{Conclusion}

24 La marge est le lieu d'une dimension réflexive sur soi-même, sur les autres, sur le monde et sa conjoncture. bell hooks (1989) définit la marge comme le point de départ d'un discours contre-hégémonique. Le quartier faisait exister un "tiers espace» (Nnaemeka 2003) : un lieu de rencontre entre les pratiques et les théories. Pour rendre compte de cet entrelacement qui rend une vie reconnaissable, l'écriture doit négocier entre les discours et les mythes. Pour raconter ailleurs ce qui a existé ici, elle est obligée de faire fiction. Elle ne doit pas seulement qualifier, elle doit renommer. Elle doit faire vivre des personnages et des lieux.

25 Ballerine est l'une d'entre elles. Je n'ai pas interviewé Ballerine. Ballerine a disparu, elle se loge dans la mémoire des anciennes qui l'ont fait vivre et dans les rêves des plus jeunes qui aimeraient la voir renaître. J'ai collecté des traces de Ballerine dans les différents entretiens menés avec les habitant-e-s du quartier et j'ai réécrit Ballerine. En incarnant les traces de ce qui a compté et de ce qui a disparu, Ballerine raconte ce qui faisait la valeur de la vie du quartier :

Les filles de Ballerine, elles sont dans les quartiers maintenant. Elles vendent par-ci, par-là. On reste tranquille. On ne sait pas ce que Dieu va faire. Parce qu'à l'époque, les filles de Ballerine sortaient avec des hauts gradés, des grands galons. Peut-être qu'ils vont vouloir revenir chercher leurs copines. On attend un jour. On attend que le président signe le contrat avec les militaires français. Alors tout le monde sautera sur ses pieds. Tout le monde 
va sortir. Ballerine va revenir. Sans conditions! C'est nous qui allons commander! (Ballerine, 2010)

\section{BIBLIOGRAPHIE}

hooks, bell. 1989. « Choosing the Margin as a Space of Radical Openness », in Gender, Space, Architecture : An Interdisciplinary Introduction, RENDELL, Jane, PENNER, Barbara et BORDEN, Iain (éds). Londres : Routledge, 203-209.

CARITE, Didier. 2009-2012. « Yakoyé et Hellani, muses oubanguiennes » Images et mémoires 23 : $14-20$.

FARGE, Arlette. 2002. «Penser et définir l'événement en histoire. Approche des situations et des acteurs sociaux » Revue Terrain 38 : p. 67-78.

NnAEMEKA, Obioma. 2004. « Nego-feminism : Theorizing, Practicing, and Pruning Africa's Way » Signs 29(2) : 357-385.

OGRIZEK, Michel. 1981. «Mami Wata, les envoûtées de la sirène. Psychothérapie collective de l'hystérie en pays Batsangui au Congo, suivie d'un voyage mythologique en Centrafrique » Cahiers de l'ORSTOM 18(4) : 433-443.

SAID, Edward. 2000. Culture et impérialisme. Paris : Fayard.

TABET, Paola. 2004. La grande arnaque. Sexualité des femmes et échange économico-sexuel. Paris :

L'Harmattan.

TonDA, Joseph. 2005 Le Souverain moderne. Le corps du pouvoir en Afrique centrale (Congo, Gabon). Paris : Karthala.

TSIBINDA, Marie-Léontine. 1987. Demain un autre jour, Paris : Silex.

YabouZA, Adrienne et Pinguilly, Yves. 2008. La défaite des mères. Paris : Oslo.

\section{NOTES}

1. Mami-Wata peuple les romans et la biographie de Marie-Léontine Tsibinda. Marie-Léontine Tsibinda, bibliothécaire et écrivain, est née en 1958 à Girard, en République Démocratique du Congo.

2. godobè, qui signifie voyou en sango, est un mot utilisé pour désigner les enfants et les jeunes qui travaillent et dorment au marché du centre-ville. Godobè est l'équivalent d'enfants des rues.

3. La notion de « droit " recouvre aussi celle du devoir.

4. gba-mûnjû, signifie littéralement en sango s'accoupler avec le blanc, et désigne les femmes qui recherchent particulièrement les blancs à Bangui, et de façon plus générale, toutes les femmes catégorisées comme prostituées. 


\section{RÉSUMÉS}

Cet article interroge le passage entre l'enquête et le texte à partir de la question des traces et de la perte. Quelle est la perte dans le texte ethnographique? De quoi le texte ethnographique peutil se faire la trace? L'article interroge le processus d'écriture d'une enquête ethnographique menée à Bangui sur un quartier d'habitation réunissant des femmes centrafricaines qui travaillaient dans les espaces nocturnes des expatriés français comme serveuses et prostituées. À travers trois figures sociales, mémorielles et mythiques rencontrées pendant l'enquête, l'article interroge les limites de l'écriture ethnographique pour transmettre dans le contexte académique les vies quotidiennes des femmes, faire mémoire et reconnaître ce qui faisait la valeur de leurs vies.

This article questions the passage between the survey and the text from the point of view of the issue of traces and the loss. What is the loss in the ethnographic text? Of which reality the ethnographic text can be the trace? The article questions the process of writing on an ethnographic survey conducted in Bangui on a neighborhood of Central African women who worked as waitresses and prostitutes for French expatriates. Through three social, memorial and mythical figures encountered during the investigation, the article questions the limits of ethnographic writing to transmit in the academic context the daily lives of women, to remember and recognize what made the value of their lives.

\section{INDEX}

Thèmes : Recherches

Mots-clés : travail du sexe, écriture ethnographique, enquête ethnographique, mémoire, République Centrafricaine

Keywords : sex work, ethnographic writing, ethnographic survey, memory, Central African Republic

\section{AUTEUR}

\section{MAGDALENA BRAND}

Magdalena Brand était doctorante en anthropologie à l'Université de Saint-Denis de 2009 à 2015, rattachée à l'équipe CSU du laboratoire CRESPPA. Elle est aujourd'hui bibliothécaire documentaliste. 